\title{
Chorea in the Elderly with Mutation Positive Polycythemia Vera: A Case Report
}

\author{
Hrishikesh Kumar, Paul Masiowski, Mandar Jog
}

Can. J. Neurol. Sci. 2009; 36:370-372

Chorea is a hyperkinetic movement disorder characterized by excessive spontaneous movements that are irregularly timed, randomly distributed and abrupt. Chorea in an elderly patient poses a diagnostic challenge. The differential diagnosis is broad and includes medication effects, vascular disorders, metabolic abnormalities, autoimmune syndromes and neurodegenerative disorders. ${ }^{1,2}$ Important causes of late onset chorea have been listed in the Table. A significant number of cases of late-onset chorea without a clear etiology are lumped together as "senile chorea". This diagnostic term may obscure a subset of cases where a specific diagnosis can be clinched with further investigations.

We present a case of new onset chorea in an elderly patient uncovering Polycythemia Vera (PV) with hematological examination positive for the characteristic Janus Kinase-2 (JAK2) mutation. Chorea was the only presenting feature in this patient, and treatment of polycythemia led to reduction of the abnormal movements significantly. We also review the association of PV and chorea and possible mechanism of causation of chorea in patients with polycythemia.

\section{Case Report}

An 82-year-old woman presented with new onset of chorea. The week before, she had noticed her tongue suddenly felt thick and heavy. Involuntary movements of the mouth, lips and tongue appeared gradually over the next few days, with subsequent involvement of the trunk and limbs. The movements were irregular, abrupt and appeared to flow from one body part to another. They were present continuously except during sleep and the patient could not suppress them voluntarily. She had no other symptoms. No memory loss or behavioral change was reported. She had no prior history of chorea, other movement or any psychiatric illness. She had a history of hypertension and had smoked one pack of cigarettes daily for the last fifty years. Her usual medications were hydrochlorothiazide, irbesartan and acetaminophen with codeine. She had no history of rheumatic fever, diabetes or stroke and had no other drug exposure. There was no family history of chorea or dementia.

On examination, she had chorea of the tongue, lips and posterior pharyngeal wall. Symmetrical choreic movements of the trunk and both upper limbs were also present. Neuropsychiatric assessment was normal. Cranial nerve examination including extra-ocular movements was normal and she was able to keep the tongue protruded for more than one minute. Tone, power, reflexes, sensation and coordination were normal, except for bilaterally diminished ankle jerks. She was moderately plethoric and had a quiet systolic murmur. She did not have cyanosis or icterus and spleen was not palpable.

Investigations were suggestive of polycythemia: RBCs $6.92 \mathrm{x}$ $10^{12} / \mathrm{L}, \mathrm{Hb} 181 \mathrm{~g} / \mathrm{L}$, Hct 56\%, WBCs $9.6 \times 10^{9} / \mathrm{L}$, and platelets $492 \times 10^{9} / \mathrm{L}$. Normal test results included TSH, calcium, sodium, creatinine, bilirubin, liver enzymes, glucose, and hemoglobin A1c. Ferritin was 17.1 (low-normal) and serum erythropoietin level was 3.2 (low). The anti-streptolysin O screen was negative. Magnetic resonance imaging brain was normal except for age related cortical atrophy. On JAK2 mutation testing, she had $\mathrm{V} 617 \mathrm{~F}$ mutation, confirming the diagnosis of PV. After consultation with a hematologist, hydroxyurea was started in the dosage of $1000 \mathrm{mg}$ per day. Two weeks later, her chorea had significantly improved and blood counts showed $\mathrm{Hb} 172 \mathrm{~g} / \mathrm{L}$, Hct $51 \%$, WBCs $6.0 \times 10^{9} / \mathrm{L}$, and platelets $247 \times 10^{9} / 1$. She has been followed up for six months and is free from chorea.

\section{Discussion}

Polycythemia Vera (PV) is a state of chronic proliferation of morphologically normal red cells as a result of clonal expansion of hematopoietic stem cells. Polycythemia Vera is strongly associated with a gain-of-function mutation in the gene for the JAK2 (V617F) that renders hematopoietic stem cells very sensitive to erythropoietin and other growth factors. ${ }^{3}$ More than $90 \%$ of patients of PV carry a V617F JAK2 mutation. ${ }^{4,5}$ Clonal hematopoiesis occurs in PV despite low serum levels of erythropoietin, differentiating it from erythrocytosis. The cardinal feature of PV is an increase in red cell mass. The hematocrit is often used as a proxy measure for red cell mass in PV. Polycythemia Vera is almost always a condition of elevated hematocrit and chronic hyperviscosity. Hyperviscosity alters the dynamics of both arterial and venous cerebral blood flow, decreasing cerebral perfusion and increasing venous stasis and distension.
From the Movement Disorder Program, London Health Sciences Centre, London
Ontario, Canada.
ReCEIVED November 17, 2008. FInAL Revisions SubMitTED JANUARY 9, 2009.
Correspondence to: : Mandar Jog, Director, Movement Disorder Program, London
Health Sciences Centre, 339, Windermere Road, London, Ontario, N6A 5A5, Canada. 


\section{Table: Important causes of late-onset chorea}

\begin{tabular}{ll}
\hline Vascular & Cerebrovascular disease(ischemic and hemorrhagic), Chronic sub-dural hematoma \\
Degenerative & Huntington's disease, Huntington disease like 1,2,3 (HDL-1,2,3), Rare cases of Spino-cerebellar Ataxia, \\
& Neuroferritinopathies. \\
Creutzfeldt Jacob disease, Acquired Immune Dificiency Syndrome, Neurosyphylis, Lyme's disease, \\
Encephalitis
\end{tabular}

These hemodynamic changes frequently manifest themselves as non-localizing neurological symptoms. At the time of diagnosis of $\mathrm{PV}, 50-80 \%$ of patients have symptoms such as headaches, dizziness, paresthesias, and blurred vision. The other frequent neurological manifestation in PV is stroke, which causes significant morbidity and mortality. Chorea is the only movement disorder described in association with PV with any consistency. The association was first described in 1909 by Umney and Bordachzi. ${ }^{6,7}$ Since then, sporadic papers, mostly case reports, have appeared in literature from time to time. In most cases, chorea was the presenting complaint and led to the diagnosis of PV. ${ }^{8-14}$ Most reported patients were older adults probably reflecting the median age of diagnosis of PV of 60 years. Majority of the reported patients of polycythemic chorea were females, despite the fact that more men have PV (male:female ratio of 3:2). ${ }^{11}$ A similar female predominance is seen in other choreic disorders with autoimmune or endocrine causes.

Most patients who develop polycythemic chorea had never experienced chorea previously. The typical clinical picture in polycythemic chorea is of generalized symmetrical chorea, with prominent involvement of the oro-facio-lingual muscles. The presentation has been likened to tardive dyskinesia. A few cases of unilateral polycythemic chorea have been described but magnetic resonance imaging has failed to demonstrate a contralateral structural lesion or any area of restricted diffusion in those cases. ${ }^{15}$ Sporadic cases of chorea associated with polycythemia and congenital cyanotic heart disease have also been reported. ${ }^{16}$ Besides hypotonia of the limbs, the neurological examination is usually normal. Mental status was preserved in the majority of the reported cases..$^{8-14}$

The course of polycythemic chorea often waxes and wanes in parallel with the hematocrit, though there is no consistent relationship between them. ${ }^{9}$ Chorea often first appears with the initial onset of PV or with a sudden exacerbation of previously well-controlled disease. ${ }^{14}$ Effective treatment of polycythemia usually improves chorea, sometimes dramatically. But the course can be independent of changes in the blood counts - chorea may persist long after the hematocrit has normalized or it may resolve spontaneously without treatment for polycythemia. Most cases do resolve fully within a few weeks or months, though some have lasted years.

Neuroimaging studies (CT scan and MRI) have not shown a consistent pattern of focal lesions in the basal ganglia in patients with polycythemic chorea and they were normal in the vast majority of cases. Even the cases of PV with hemichorea had normal neuro-imaging. ${ }^{15}$ Results of cerebral blood flow analysis in cases of PV with chorea have been conflicting. A recent study using subtraction SPECT with co-registered MRI did not find any reduction in blood flow. ${ }^{17}$

Only limited data regarding neuropathology in PV with chorea are available. Prominent venous congestion in brain parenchyma and dura mater was described in some of the cases. ${ }^{18}$ Focal demyelination, described in rare instances does not sufficiently explain the clinical picture of polycythemic chorea. A similar but more severe pattern of multifocal perivenous demyelination was described in a patient with PV who did not have chorea. ${ }^{19}$ By inference, it can be assumed that polycythemic chorea is a syndrome without a specific pathological correlate.

The pathophysiology of polycythemic chorea is not well understood. The syndrome has been attributed to neo-striatal hypoperfusion related to hyperviscosity. ${ }^{11}$ But the relationship between hyperviscosity, hypo-perfusion and chorea is tenuous. Fewer than $1 \%$ of patients with PV develop chorea, even though almost all of them have symptoms of hyperviscosity, neurological or otherwise. Patients who develop chorea with PV need not have an especially high hematocrit and their symptoms may run its course independent of changes in the blood counts. Severe, symptomatic hyperviscosity is also seen in Waldenstrom's macroglobulinemia and multiple myeloma, but chorea has not been reported in association with these 
conditions. The difference may lie in the fact that hyperviscosity in the paraproteinemias occurs because of increased protein content in the plasma fraction of whole blood, as opposed to the increased cellular fraction seen in polycythemia. Other hypotheses including microvascular changes and polycythaemic increase of dopamine-laden platelets releasing excess of dopamine in the neostriatum are still speculative. ${ }^{11,20,21}$.

Polycythemic chorea can be treated symptomatically with neuroleptics or benzodiazepines. Effective treatment of the underlying polycythemic state is crucial, as many cases have shown rapid improvement and resolution of chorea once the hematocrit reduces. Aspirin and phlebotomy have been recommended in low risk cases and hydroxyurea in high risk cases. ${ }^{4,22}$ Novel therapies in the form of JAK2 V617F inhibitors are being developed and hold promise as future treatment options. $^{23}$

The present case has hematological attributes of PV and positive JAK2 mutation. The sole presenting feature in this case was generalized chorea and no radiological correlate could be found on MRI of brain. Treatment with hydroxyurea led to marked reduction in chorea and there was associated improvement in hematological parameters. Though the association between PV and chorea has long been known, the present case is the first report of JAK2 mutation positive PV presenting with new onset generalized chorea. JAK2 mutation testing is rapidly becoming an important diagnostic tool for myeloproliferative disorders like PV. Detection of this mutation in the present case strengthens the historical evidence regarding association of chorea with Polycythemia Vera. We will be inclined to recommend JAK2 mutation test in elderly patients with undiagnosed chorea. Furthermore, it can be speculated that new treatment options like JAK2 V617 F inhibitors might be of value in treating polycythemic chorea in the near future.

\section{REFERENCES}

1. Wild EJ, Tabrizi SJ. The differential diagnosis of chorea. Pract Neurol. 2007 Nov;7(6):360-73.

2. Cardoso F, Seppi K, Mair KJ, Wenning GK, Poewe W. Seminars on choreas. Lancet Neurol. 2006 Jul;5(7):589-602.

3. Kilpivaara O, Levine RL. JAK2 and MPL mutations in myeloproliferative neoplasms: discovery and science. Leukemia. 2008 Oct;22(10):1813-7.

4. Finazzi G, Barbui T. The treatment of polcythemia vera: an update in the JAK2 era. Intern Emerg Med. 2007 March;2(1):13-8.

5. Tefferi A, Thiele J, Orazi A, Kvasnicka HM, Barbui T, Hanson CA, et al. Proposals and rationale for revision of the World Health Organization diagnostic criteria for polycythemia vera, essential thrombocythemia, and primary myelofibrosis: recommendations from an ad hoc international expert panel. Blood. 2007 Aug 15;110(4):1092-7.
6. Umney WF. Notes on a fatal case of splenomegalic polycythemia. Lancet. 1909;I:1243-5.

7. Bordachzi P. Chorea und polycythemia vera. Prag Med Wochr. 1909;34:253.

8. Cohen AM, Gelvan A, Yarmolovsky A, Djaldetti M. Chorea in polycythemia vera: a rare presentation of hyperviscosity. Blut. 1989 Jan;58(1):47-8.

9. Voiculescu V, Ionescu DA, Lazar G, Alecu C, Predescu C, Popa C, et al. Two cases of choreatic syndrome caused by polycythemia vera. Eur Neurol. 1979;18(2):96-100.

10. Mas JL, Gueguen B, Bouche P, Derouesné C, Varet B, Castaigne P. Chorea and polycythaemia. J Neurol. 1985;232(3):169-71.

11. Bruyn GW, Padberg G. Chorea and polycythaemia. Eur Neurol. 1984;23(1):26-33.

12. Morré HH, van Woerkom TC, Endtz LJ. A case of chorea due to polycythaemia vera. Clin Neurol Neurosurg. 1982;84(2):125-30.

13. Borg-Costanzi JM, Mohr PD, Lewis D. Primary polycythaemia presenting with chorea. Postgrad Med J. 1981 Apr;57(666): 228-31.

14. Nazabal ER, Lopez JM, Perez PA, Del Corral PR. Chorea disclosing deterioration of polycythaemia. Postgrad Med J. 2000 Oct; 76:658-9.

15. Midi I, Dib H, Köseoglu M, Afsar N, Günal DI. Hemichorea associated with polycythaemia vera. Neurol Sci. 2006 Dec;27 (6):439-41.

16. Edwards PD, Prosser R, Wells CE. Chorea, polycythaemis, and cyanotic heart disease. J Neurol Neurosurg Psychiatry. 1975 Aug;38(8):729-39.

17. Kim W, Kim JS, Lee KS, Kim YI, Park CW, Chung YA. No evidence of perfusion abnormalities in the basal ganglia of a patient with generalized chorea-ballism and polycythaemia vera: analysis using subtraction SPECT co-registered to MRI. Neurol Sci. 2008 Oct;29(5):351-4.

18. Kotner LM, Tritt JH. Chorea complicating polycythemia vera: report of a case. Ann Int Med.1942;17: 544-8.

19. D'agostino AN, Pease GL, Kernohan FW. Cerebral demyelination associated with polycythemia vera. J Neuropathol Exp Neurol. 1963 Jan;22:138-47.

20. Landolfi R, Di Gennaro L, Falalngo A. Thrombocytosis in myeloproliferative disorders: pathogenetic facts and speculation. Leukemia. 2008 Nov;22(11):2020-8.

21. Landolfi R, Cipriani MC, Novarese L. Thrombosis and bleeding in polycythemia vera and essential thrombocythemia: pathogenic mechanism and prevention. Best Pract Res Clin Haematol. 2006;19(3):617-33.

22. Finazzi G, Barbui T. Evidence and expertise in the management of polycythemia vera and essential thrombocythemia. Leukemia. 2008 Aug;22(8):1494-502.

23. Pardanani A, Hood J, Lasho T, Levine RL, Martin MB, Noronha G, et al. TG101209, a small molecule JAK2-selective kinase inhibitor potently inhibits myeloproliferative disorder-associated JAK2 V617F and MPL515L/K mutations. Leukemia. 2007;21: 1658-68. 